\title{
L'historiographie des Amérindiens au Canada : quelques données et commentaires portant sur les directions de la recherche et sur les travaux en cours
}

\section{Jan Grabowski}

Volume 53, numéro 4, printemps 2000

Histoire des Premières Nations : nouvelles lectures et nouveaux problèmes

URI : https://id.erudit.org/iderudit/005454ar

DOI : https://doi.org/10.7202/005454ar

Aller au sommaire du numéro

Éditeur(s)

Institut d'histoire de l'Amérique française

ISSN

0035-2357 (imprimé)

1492-1383 (numérique)

Découvrir la revue

Citer cet article

Grabowski, J. (2000). L'historiographie des Amérindiens au Canada : quelques données et commentaires portant sur les directions de la recherche et sur les travaux en cours. Revue d'histoire de l'Amérique française, 53(4), 552-560. https://doi.org/10.7202/005454ar 


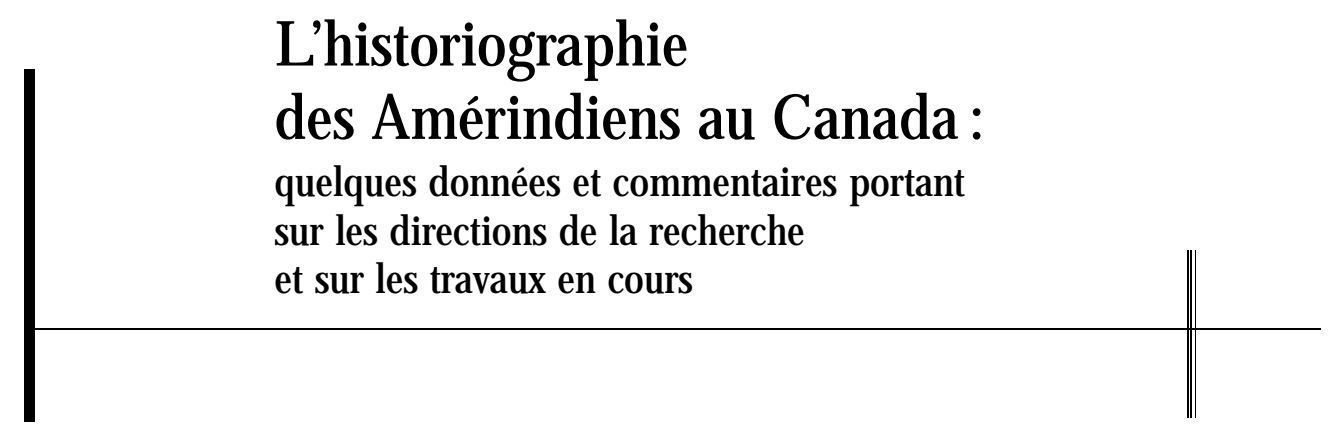

JAN GRABOWSKI

Département d'histoire

Université d'Ottawa

A PRÉSENTE ÉTUde a pour but de faire la lumière sur le développement des recherches amérindiennes au Canada pendant les quinze dernières années. L'accélération des changements institutionnels et politiques concernant les Autochtones a provoqué un intérêt accru dans I'opinion publique et aussi chez les chercheurs dans les différentes disciplines touchant à l'histoire et à l'expérience amérindiennes au Canada. Cet intérêt s'est traduit par une véritable multiplication de nouvelles publications et de nouveaux projets de recherche. II y a cependant peu d'informations sur les tendances de cette recherche.

Étant donné le nombre de publications qui voient le jour actuellement, que ce soit en français ou en anglais, il est devenu très difficile au chercheur de se tenir parfaitement informé. Les données et les commentaires présentés ici devraient faciliter le futur débat sur la question de la place des études amérindiennes dans I'historiographie canadienne.

Dans son article publié en 1986, I'anthropologue Bruce Trigger a fait état de la condition des études sur les Premières Nations au Canada'. Ayant tracé les grandes lignes du développement de la discipline, Trigger s'est interrogé sur l'avenir de ce champ d'étude. Selon lui, bien que la

1. Bruce G. Trigger, «The Historian's Indian: Native Americans in Canadian Historical Writing from Charlevoix to the Present», Canadian Historical Review, 67,3 (1986) : 315-342. 
discipline ait connu un essor remarquable, le «courant dominant» historiographique ne tenait toujours pas compte des recherches amérindiennes. Une quinzaine d'années après la publication de cet article, il semble opportun de s'interroger à nouveau sur le même thème. La discipline s'est en effet développée de manière significative et elle a emprunté plusieurs chemins. Les peuples autochtones constituent aujourd'hui un des champs d'étude les plus «éclectiques», mobilisant à la fois les méthodes des sciences sociales et celles des sciences humaines Le domaine est ouvert autant aux anthropologues, aux spécialistes du droit, aux sociologues et aux archéologues qu'aux historiens

Pour s'en rendre compte, il suffit de faire état des articles et thèses consacrés aux Premières Nations depuis une douzaine d'années Nous avons basé notre étude sur 400 articles publiés entre 1988 et 1996 et 179 thèses soutenues entre 1986 et 1997. Les articles, parus dans les périodiques répertoriés par l'index America History and Life, traitent de divers aspects de I'histoire autochtone au Canada. La liste des thèses en histoire a été élaborée à partir des «Rapports» de la Société historique du Canada. Nous examinerons d'abord les articles et ensuite les thèses

\section{LES A R T ICLES}

Nous avons rassemblé les informations relatives aux articles dans les tableaux 1,2 et 3 , que nous avons divisés chaque fois en trois périodes (1988-1990, 1991-1993 et 1994-1996), afin de mieux saisir les changements de tendances de la recherche.

À l'évidence, on a assisté, ces demières années, à un double phénomène: d'une part, l'accroissement de la production scientifique et, d'autre part, sa spécialisation constante. Deux sujets de recherche ont eu priorité: la politique indienne de l'État et l'autonomie gouvernementale des Amérindiens. Si on ne peut que se féliciter de voir plusieurs études portant sur des questions aussi importantes, on ne peut par contre que regretter l'appauvrissement graduel des autres secteurs de la recherche. Le pourcentage des travaux consacrés à I'histoire militaire connait une diminution continue. Entre 1988 et 1996, il est passé de $20 \%$ à $8 \%$ de la production historique. II en va de même pour I'histoire sociale, culturelle et pour celle de la religion.

La couverture géographique de la recherche indique clairement que les chercheurs abordent le plus souvent des questions portant sur la totalité du pays, des questions de nature théorique et générale. Ce genre d'exercice a pour objet habituel la politique indienne de l'État. 
t a blea u 1

Sujets abordés

\begin{tabular}{lrrrc}
\hline & 1988-1990 & 1991-1993 & 1994-1996 & $\begin{array}{c}\text { Total } \\
\text { 1988-1996 }\end{array}$ \\
\hline Militaire/ Diplomatique & 23 & 21 & 9 & 53 \\
Culture & 23 & 29 & 11 & 63 \\
Économie/ Commerce/ Travail & 14 & 15 & 6 & 35 \\
Politique indienne et & & & & \\
autonomie go uvernementale & 53 & 64 & 73 & 190 \\
Éducation & 2 & 3 & 4 & 9 \\
Histoire sociale & 14 & 18 & 9 & 41 \\
Religion & 10 & 7 & 8 & 25 \\
Femmes & 7 & 10 & 12 & 29 \\
Art et littérature & 21 & 14 & 9 & 44 \\
Archéologie & 3 & 6 & 3 & 12 \\
Autres & 11 & 20 & 19 & 50 \\
\hline Total* & 181 & 207 & 163 & 551 \\
Nombre total d'articles & 132 & 148 & 121 & 401 \\
\hline
\end{tabular}

* Certains articles touchent à plus d'un sujet.

t a blea $u 2$

\section{Les régions étudiées}

\begin{tabular}{lcccc}
\hline & 1988-1990 & 1991-1993 & 1994-1996 & Total \\
\hline O uest & 31 & 40 & 39 & 110 \\
O ntario/ Q uébec & 26 & 32 & 21 & 79 \\
Est & 7 & 8 & 6 & 21 \\
N ord & 21 & 14 & 13 & 48 \\
Canada & 47 & 54 & 42 & 143 \\
\hline Total & 132 & 148 & 121 & 401 \\
\hline
\end{tabular}


t a bleau 3

Les époques couvertes par la recherche

\begin{tabular}{lcccc}
\hline & 1988-1990 & 1991-1993 & 1994-1996 & Total \\
\hline A vant contact & 5 & 3 & 39 & 11 \\
x vile-xviie siècles & 11 & 18 & 10 & 39 \\
x viï siècle & 15 & 26 & 18 & 59 \\
$1801-1850$ & 17 & 21 & 15 & 53 \\
$1851-1900$ & 41 & 42 & 42 & 118 \\
$1901-1950$ & 31 & 31 & 41 & 103 \\
$1951-1996$ & 65 & 77 & 73 & 215 \\
\hline Total* & 185 & 218 & 238 & 598 \\
N ombre total d'articles & 132 & 148 & 121 & 401 \\
\hline
\end{tabular}

* Certains articles concernent plus d'une période.

II semble (mais il faudrait faire des recherches plus poussées) qu'un nombre considérable d'ouvrages historiques - articles et livres - dans le domaine soient étroitement liés aux études animées et commanditées par les différents organismes judiciaires ou parajudiciaires. Cela n'est pas surprenant, étant donné le nombre de revendications territoriales et autres causes impliquant les Amérindiens qui traînent devant les tribunaux depuis plusieurs années. Nous devons donc nous interroger sur le rôle et sur l'influence que la recherche "partisane», c'est-à-dire entreprise pour répondre à une commande, exerce sur l'ensemble de I'historiographie amérindienne au Canada.

\section{I es thèses}

Étant donné que l'avenir de la discipline est étroitement lié aux intérêts des jeunes chercheurs qui commencent leur carrière, un survol des thèses s'impose. Nous avons trouvé 179 thèses (maîtrise et doctorat) concernant I'histoire des Autochtones du Canada et soutenues dans les départements d'histoire des universités canadiennes entre 1986 et 1997 . Il faut souligner que les tableaux ci-dessous ne tiennent pas compte de la multitude de thèses abordant la thématique amérindienne dans les disciplines autres que l'histoire.

2. Étant donné que les thèses portent souvent sur plus d'un sujet ou sur plus d'une période, les totaux dans les tableaux qui suivent dépassent souvent le nombre des thèses retenues pour cette étude. 
t a b l ea u 4

Thèses par année

\begin{tabular}{lr}
\hline 1986 & 9 \\
1987 & 6 \\
1988 & 5 \\
1989 & 7 \\
1990 & 11 \\
1991 & 10 \\
1992 & 17 \\
1993 & 12 \\
1994 & 24 \\
1995 & 31 \\
1996 & 22 \\
1997 & 25 \\
\hline Total & 179 \\
\hline
\end{tabular}

Comme l'indique le tableau 4, le nombre de thèses connaît une poussée en 1990 et une autre encore plus importante à partir de 1994, avec un sommet de 31 thèses soutenues en 1995. II existe donc une tendance générale qui favorise le développement des études sur les Autochtones. Tendance que confirme la part prise par les thèses «amérindiennes» dans la totalité des thèses portant sur un sujet canadien: de 1986 à 1989, cette part était de 4,2\%; de 1990 à 1993, de 5,5\%; et de 1994 à 1997, de 12,4\%. En 1997, la production historique relative aux Autochtones représentait près de $13 \%$ de ce qui a été consacré aux autres sujets en histoire canadienne. Cela constitue sans doute une baisse par rapport à l'année-record 1995, quand les thèses en histoire amérindienne franchissaient le seuil de $15 \%$. Néanmoins la croissance est significative. Les études amérindiennes constituent désomais un secteur appréciable de la recherche historique au Canada.

Cette poussée est accompagnée d'un phénomène intéessant. Dans un article paru en 1984 , John A. Price estimait que seulement $13 \%$ des thèses en histoire portant sur les Amérindiens avaient été écrites par des

3. John A. Price, «A Critical Analysis of Graduate Theses in Native Studies», The Canadian Journal of Native Studies/ La Revue canadienne des études autochtones, 6,1 (1984) : 141-146. 
femmes. À la lumière des données récentes nous y voyons un progrès important: aujourd'hui, les femmes représentent plus de la moitié ( $51 \%$ ou 91 sur 179 personnes) de tous les diplômés en histoire des Autochtones.

Notons toutefois que ces changements n'ont pas apporté de modifications majeures dans la répartition des thèses selon les régions étudiées et les sujets retenus. Sur le plan géographique, John A. Price s'inquiétait, dans l'article précédemment mentionné, de la «nationalisation » et de la régionalisation des études amérindiennes Selon lui, la vaste majorité des spécialistes dans le domaine ne s'aenturaient guère au-delà des frontières canadiennes en limitant leurs recherches aux Premières Nations d'ici. En 1984, $97 \%$ des thèses produites dans les universités canadiennes concernaient uniquement les Amérindiens vivant sur le sol canadien. Nous sarons, cependant, que le monde aborigène transcendait les frontières politiques d'aujourd'hui. Or, comme le précise le tableau 5, les régions étudiées n'ont guère changé depuis 1984.

II existe toujours un nombre considérable d'études portant sur l'ensemble du territoire canadien: le plus souvent, il s'agit d'études théoriques. II faut indiquer aussi le peu de travaux qui portent sur l'expé rience autochtone dans les provinces maritimes: on en compte seulement sept pendant toute la période cibée.

t a bleau 5

Thèses selon les régions étudiées

\begin{tabular}{lr}
\hline Canada & 57 \\
O ntario & 26 \\
Q uébec & 23 \\
Colombie-Britannique & 18 \\
N ord & 15 \\
Manitoba & 14 \\
Alberta & 13 \\
Autres & 18 \\
\hline Total* & 184 \\
N ombre total de thèses & 179 \\
\hline
\end{tabular}

* Certaines thèses concer nent plus d'une région. 
t a bleau 6

Thèses selon les sujets

\begin{tabular}{lr}
\hline Politique indienne et & \\
l'autonomie gouvemementale & 59 \\
Culture & 20 \\
Société & 20 \\
Histoire militaire et diplomatique & 17 \\
Religion & 14 \\
Éducation & 12 \\
Économie & 11 \\
Femmes & 11 \\
Autres* & 33 \\
\hline Total** & 197 \\
Nombre total de thèses & 179 \\
\hline
\end{tabular}

* Parmi les «autres sujets», nous avons retenu Art, Justice, Préhistoire, Muséologie. La catégorie « Éducation » concerne surtout les études portant sur les écoles résidentielles ou industrielles.

** Certaines thèses traitent de plus d'un sujet.

Remarques comparables en ce qui concerne les sujets choisis Comme dans le cas des articles, le groupe le plus important de thèses aborde l'autonomie gouvernementale et la politique indienne de l'État canadien. Cette catégorie domine le champ et sembe étroitement liée aux événements politiques contemporains. De 1986 à 1997, quelque 59 travaux, portant uniquement ou entre autres sur cette question, ont vu le jour. Les autres thèses ont touché en priorité aux thèmes consacrés à la culture, à la société, à l'histoire militaire et diplomatique, et à la religion. Au fil des années, ces thèmes, avec la politique indienne et l'autonomie gouvernementale, ont prévalu parmi les sujets de thèse. La croissance du nombre de thèses n'a pas remis en cause la prépondérance de ces thèmes, et la forte avance de la politique et de l'autonomie sur les auties sujets est demeurée la même.

Pour sa part, la répartition chronologique des thèses confirme le poids des débats politiques actuels dans la recherche historique. Le tableau 7 comprend les thèses dont le cadre chronologique porte sur plusieurs périodes. De toute évidence, les étudiants diplômés focalisent leurs recherches surtout sur la période après la Confédération (168 travaux sur 293). Cette tendance devient encore plus évidente si nous ne retenons 
tableau 7

Thèses selon la période étudiée

\begin{tabular}{lrc}
\hline Préhistoire & 13 & $(8)^{*}$ \\
xvie-xviie siècles & 22 & $(4)$ \\
xviiie siècle & 38 & $(6)$ \\
$1801-1850$ & 52 & $(8)$ \\
$1851-1900$ & 53 & $(11)$ \\
$1901-1950$ & 56 & $(14)$ \\
$1951-1998$ & 59 & $(34)$ \\
\hline Total & 293 & $(85)$ \\
Nombre total de thèses & 179 & \\
\hline
\end{tabular}

* Les chiffres entre parenthèses indiquent les thèses touchant à une seule période.

que les thèses portant sur une seule période. N on seulement lexx ${ }^{\mathrm{e}}$ siècle devient la période la plus fouillée, mais les étudiants affichent un intérêt tout particulier pour les dernières décennies.

conclusion

Alors qu'il y a une vingtaine d'années les recherches relatives aux Autochtones au Canada demeuraient l'exception, aujourd'hui, la situation semble plus favorable. Chaque année apporte au moins quarante nouvelles publications dans les revues savantes (et le nombre est certainement plus élevé puisque nous n'avons pas examiné tous les périodiques). II est certain - si on pouvait étendre cette étude au-delà des articles et des thèses - que le même phénomène se reproduirait dans le cas des monographies.

Mal gré cette abondance, il faut constater que de nombreux aspects de I'histoire amérindienne demeurent relativement inexplorés. La prédominance de la recherche axée sur la politique indienne et sur l'autonomie gouvernementale a relégué au deuxième plan les travaux en histoire religieuse, sociale et militaire des aborigènes. L'importance démesurée accordée à la période la plus récente a condamné aux marges de la discipline les études portant sur l'expérience coloniale. Dans le contexte géographique, le plus frappant est le formidable écart entre la multitude des travaux visant les provinces de l'Ouest et l'absence d'un intérêt comparable dans l'Est du pays. 
L'autre question, qui sort du cadre de cette étude mais dont l'importance est indéniable, concerne le rôle du système judiciaire dans l'enquête historique. À plusieurs reprises les tribunaux ont «déterminé» la nature des phénomènes historiques Selon la Cour suprême du Canada (Reinec. Sioui, 1990) la proclamation du général Murray de 1760 doit, en réalité, être considérée comme un traité avec les Autochtones. Récemment, en septembre 1999, la même cour s'est prononcée sur l'importance et la valeur du traité de 1760-1761 entre la Couronne et les Mi'kmaq (Reine c. M arshall, 1999). Les décisions de la Cour mettent-elles fin aux débats historiques? Les preuves du contraire constituent-elles un outrage au tribunal? Il est certain, en tout cas, que la cohabitation entre les historiens et les avocats est là pour rester, ce qui oblige les premiers à affronter des questions méthodologiques et éthiques pour le moins singulières. 